\title{
Role of AUF1 in modulating the proliferation, migration and senescence of skin cells
}

\author{
DAOJIANG YU ${ }^{1,2^{*}}$, XIAOQIAN LI ${ }^{1 *}$, ZHENYU WANG $^{3 *}$, SHENG JIANG $^{2}$, TAO YAN $^{1}$, \\ KAI FANG ${ }^{2}$, YUHONG SHI ${ }^{2}$, ZHIQIANG JIANG ${ }^{2}$ and SHUYU ZHANG ${ }^{1,2,4}$
}

\author{
${ }^{1}$ West China School of Basic Medical Sciences and Forensic Medicine, Sichuan University, \\ Chengdu, Sichuan 610041; ${ }^{2}$ Department of Surgery, Second Affiliated Hospital of Chengdu Medical College, \\ China National Nuclear Corporation 416 Hospital, Chengdu, Sichuan $610051 ;{ }^{3}$ School of Radiation Medicine \\ and Protection, Soochow University, Suzhou, Jiangsu 215123; ${ }^{4}$ Department of Oncology, The Affiliated \\ Changzhou No. 2 People's Hospital of Nanjing Medical University, Changzhou, Jiangsu 213100, P.R. China
}

Received June 30, 2021; Accepted October 11, 2021

DOI: $10.3892 /$ etm.2021.10967

\begin{abstract}
AU-rich element RNA-binding factor 1 (AUF1) is a classical RNA-binding protein. AUF1 influences the process of development, apoptosis and tumorigenesis by interacting with adenylate-uridylate rich element-bearing mRNAs. Human skin is the largest organ of the body and acts as a protective barrier against pathogens and injuries. The aim of the present study was to explore the function and potential molecular pathways of AUF1 in human skin cells. AUF1 was overexpressed in human keratinocyte $\mathrm{HaCaT}$ cells and human skin fibroblast WS1 cells using adenoviruses and silenced using lentiviruses. AUF1 overexpression facilitated cell proliferation, whereas AUF1 knockdown induced the opposite effect. AUF1 reduced apoptosis but did not affect cell cycle progression. Forced AUF1 expression promoted the migration of human skin cells, as demonstrated by a scratch wound healing assay. Cell senescence was alleviated in AUF1-overexpressing skin cells, while AUF1 knockdown increased cell senescence. WS1 cells with AUF1 overexpres-
\end{abstract}

Correspondence to: Dr Zhiqiang Jiang, Department of Surgery, Second Affiliated Hospital of Chengdu Medical College, China National Nuclear Corporation 416 Hospital, 4, Section 4, Second Ring Road North, Chengdu, Sichuan 610051, P.R. China

E-mail: 1137385122@qq.com

Professor Shuyu Zhang, West China School of Basic Medical Sciences and Forensic Medicine, Sichuan University, 17 People's South Road, Chengdu, Sichuan 610041, P.R. China

E-mail: zhang.shuyu@hotmail.com

*Contributed equally

Abbreviations: RBPs, RNA-binding proteins; miRNAs, microRNAs; AUF1, AU-rich element RNA binding protein 1; ESCC, esophageal squamous cell carcinoma; HCC, hepatocellular carcinoma; RNA-Seq, RNA-sequencing

Key words: skin, AUF1, migration, senescence, apoptosis sion and silencing were used for RNA-sequencing and Kyoto Encyclopedia of Genes and Genomes-based pathway analysis to identify AUF1-affected mRNAs. A total of 18 mRNAs (eight mRNAs with positive associations and 10 mRNAs with negative associations) revealed consistent associations with both AUF1 overexpression and silencing. Enriched pathways associated with AUF1 expression included 'MAPK', 'cell adhesion molecules', 'proteasome', 'cellular senescence' and 'TGF- $\beta$ signaling', indicating a complex regulatory network. Overall, the results of the present study revealed that AUF1 is involved in the proliferation, migration and senescence of skin cells in vitro and may be a potential target for cosmetic and disease treatment of skin.

\section{Introduction}

In mammalian cells, post-transcriptional gene regulation involves multistep processes, including precursor mRNA (pre-mRNA) splicing and mRNA export, storage, stability and translation. Post-transcriptional gene regulation is substantially governed by two types of factor: RNA-binding proteins (RBPs) and microRNAs (miRNAs/miRs) (1). AU-rich element RNA binding protein 1 (AUF1), also known as heterogeneous nuclear ribonucleoprotein $\mathrm{D}$, is one of the most extensively characterized RBPs and primarily functions as an mRNA destabilizing factor (2).

A total of four isoforms of AUF1 arising from differential exon splicing of a single pre-mRNA have been identified and named according to their apparent molecular masses (p37, p40, p42 and p45) (2-4). AUF1 degrades diverse groups of transcripts, including regulators of the cell cycle and apoptosis, inflammatory mediators and cytokines and proto-oncogenes; thus, AUF1 influences oncogenic processes and inflammatory diseases $(5,6)$. For example, AUF1 in esophageal squamous cell carcinoma (ESCC) tumor tissues exhibits high expression, while knockdown of AUF1 causes markedly reduced proliferation and enhanced cell apoptosis in Eca-109 cells (7). IL-10, an important anti-inflammatory cytokine, exerts its action by inhibiting proinflammatory cytokine production by mononuclear phagocytes (8). The AUF1 isoform 
p40 plays a positive role in regulating lipopolysaccharide exposure-induced expression of IL-10 mRNA and protein in human promonocytic leukemia cells (8).

AUF1 may also be involved in thyroid carcinoma progression. A previous study has revealed that thyroid tissues have revealed increased cytoplasmic expression of AUF1 in malignant tissues (9). In hepatocellular carcinoma (HCC), AUF1 suppresses the expression of liver-specific miR-122, an established tumor suppressor miRNA, while knockdown of AUF1 promotes HCC cell death (10). AUF1 delays the senescence of vascular endothelial cells, indicating its influences on delaying aging and prolonging life (11). AUF1 was also revealed to involve the antioxidant system through regulation of superoxide dismutase 1 expression in human cancer cells (12).

Skin is the largest organ of the human body and acts as a protective barrier against pathogens and injuries (13). Mice lacking AUF1 have been demonstrated to develop chronic dermatitis, underscoring the role of AUF1 in the complex inflammatory response in skin (14). However, to the best of our knowledge, the role of AUF1 in human skin homeostasis is still unclear. The present study explored the function of AUF1 in human skin cells and characterized its downstream mRNAs by RNA-sequencing (RNA-Seq).

\section{Materials and methods}

Cell culture. Human immortalized keratinocyte HaCaT cells and human embryonic dermal fibroblast WS1 cells were purchased from the American Type Culture Collection. They were both cultured in DMEM (cat. no. D0822; Sigma-Aldrich; Merck KGaA) supplemented with $10 \%$ fetal bovine serum (FBS; cat. no. 04-001-1A; Biological Industries), $100 \mathrm{U} / \mathrm{ml}$ streptomycin and $100 \mathrm{U} / \mathrm{ml}$ penicillin (cat. no. 15140122; Gibco; Thermo Fisher Scientific, Inc.) at $37^{\circ} \mathrm{C}$ in a $5 \% \mathrm{CO}_{2}$ atmosphere.

Cell infection. To explore the function of AUF1 in skin cells, overexpression and RNA interference experiments were carried out. AUF1 complementary (c)DNA was obtained from OriGene Technologies, Inc. (cat. no. SC107836). AUF1 cDNA was subcloned into the pHBAD-EF1-mcs-CMV vector (Hanbio Biotechnology Co., Ltd.) for adenovirus construction. Packaging and purification of adenovirus overexpressing AUF1 (Ad-AUF1) and negative control adenovirus (Ad-NC) were carried out by Hanbio Biotechnology Co., Ltd. AUF1 cDNA was also subcloned into the pHBLV-U6-ZsGreen-Puro vector for lentivirus construction. Lentiviruses targeting AUF1 [named short hairpin (sh)AUF1-1 and shAUF1-2, respectively] and a scrambled negative control lentivirus (sh-NC) were purchased from Hanbio Biotechnology Co., Ltd. The target sequences against AUF1 are listed in Table SI. A mock-infected control group (without vector) was also constructed to demonstrate the background expression value.

$\mathrm{HaCaT}$ and WS1 cells were cultured in a six-well culture plate. After reaching 70\% confluence, the cells were both infected with adenoviruses and lentiviruses at a multiplicity of infection of 20 with DMEM free-serum for $6 \mathrm{~h}$ at $37^{\circ} \mathrm{C}$ in $5 \% \mathrm{CO}_{2}$. After $48 \mathrm{~h}$, the cells were harvested for further experiments, and the efficiency of infection was determined by reverse transcription-quantitative PCR (RT-qPCR) and western blotting.

RNA preparation and $R T-q P C R$. Total RNA of HaCaT and WS1 cells was extracted using TRIzol ${ }^{\circledR}$ (cat. no. 15596018; Thermo Fisher Scientific, Inc.), and the absorbance values at 230, 260 and $280 \mathrm{~nm}$ were measured to determine the purity and density of the RNA. Subsequently, $1 \mu \mathrm{g}$ of total RNA from each sample was used to synthesize single-stranded cDNA with a PrimeScript ${ }^{\mathrm{TM}}$ RT reagent kit (cat. no. RR047A; Takara Bio, Inc.). The reaction was performed at $37^{\circ} \mathrm{C}$ for $15 \mathrm{~min}$ followed by $85^{\circ} \mathrm{C}$ for $5 \mathrm{sec}$. cDNA was amplified via qPCR with TB Green ${ }^{\circledast}$ dye (cat. no. RR420S Takara Bio, Inc.) on a Prism 7500 real-time PCR machine (Applied Biosystems; Thermo Fisher Scientific, Inc.). The thermocycling conditions included an initial denaturation step of $95^{\circ} \mathrm{C}$ for $60 \mathrm{sec}$, followed by 40 cycles of amplification at $95^{\circ} \mathrm{C}$ for $15 \mathrm{sec}$ and then annealing at $60^{\circ} \mathrm{C}$ for $30 \mathrm{sec}$. The $\beta$-actin gene was used to normalize the relative fold changes of the target genes (15). The details of the primer sequences are listed in Table SII.

Western blotting. HaCaT and WS1 cells were harvested and lysed in RIPA lysis buffer (containing $50 \mathrm{mM}$ Tris- $\mathrm{HCl}$, pH 7.4, $150 \mathrm{mM} \mathrm{NaCl}, 1 \%$ Triton X-100, $1 \%$ sodium deoxycholate and $0.1 \%$ SDS) containing protease inhibitor cocktail (Sigma-Aldrich; Merck KGaA) for $30 \mathrm{~min}$ at $4^{\circ} \mathrm{C}$. Protein concentration was subsequently measured using a BCA Protein Assay kit (cat. no. P0012; Beyotime Institute of Biotechnology). Protein $(50 \mu \mathrm{g} / \mathrm{lane})$ from each lysate was fractionated by $10 \%$ SDS-PAGE and transferred to polyvinylidene difluoride membranes (MilliporeSigma). After blocking with 5\% non-fat milk in PBS and 0.1\% Tween-20 for $1 \mathrm{~h}$ at room temperature, the membranes were blotted with AUF1 (cat. no. ab282018; 1:1,000) and GAPDH (cat. no. ab181602; 1:50,000) antibodies overnight at $4^{\circ} \mathrm{C}$. After washing four times with PBST, the membranes were incubated with horseradish peroxidase-conjugated goat anti-rabbit IgG (cat. no. A21020; 1:10,000; Abbkine Scientific, Co., Ltd.) secondary antibodies for $2 \mathrm{~h}$. Following washing in PBST, the protein-bound antibodies were detected using an enhanced chemiluminescence stable peroxide solution (cat. no. DC10100; PointBio). All protein bands were visualized by a FluroChem MI imaging system (Alpha Innotech Corporation) at room temperature. Densitometry was performed using Image J Java 1.8.0-172 software (National Institutes of Health).

Cell viability and proliferation assay. After infection, cell viability was assayed using a Cell Counting Kit- 8 assay (CCK-8; cat. no. CK04; Dojindo Laboratories, Inc.). Cells were seeded in a 96-well plate $\left(1 \times 10^{4}\right.$ cells/well) and cultured for 24 and $48 \mathrm{~h}$ at $37^{\circ} \mathrm{C}$ in a $\mathrm{CO}_{2}$ incubator. After $10 \mu \mathrm{l}$ of CCK- 8 solution was added to each well, the cells were incubated for another $2 \mathrm{~h}$. Absorbance values were determined at $450 \mathrm{~nm}$ using a microplate reader (Bio-Rad Laboratories, Inc.).

To measure the proliferation of infected skin cells, an EdU incorporation assay was performed by an EdU-based cell proliferation kit (cat. no. C0071; Beyotime Institute of Biotechnology) according to the manufacturer's instructions after $48 \mathrm{~h}$ of infection. In brief, $\mathrm{HaCaT}$ and WS1 cells at $50-70 \%$ confluence were incubated with $50 \mu \mathrm{M}$ EdU for $2 \mathrm{~h}$ 
at $37^{\circ} \mathrm{C}$. Subsequently, the cells were fixed with $4 \%$ paraformaldehyde for $15 \mathrm{~min}$ at room temperature, permeabilized with $0.3 \%$ Triton X-100 and washed with phosphate-buffered saline (PBS). The cell nuclei were stained with $5 \mu \mathrm{g} / \mathrm{ml}$ Hoechst 33342 for $30 \mathrm{~min}$ at room temperature. A florescence microscope (Olympus Corporation; magnification, x20) was used to examine the cells.

Colony formation assay. HaCaT and WS1 cells infected with the indicated virus were seeded in $60-\mathrm{mm}$ petri dishes at low density (1,000 cells/dish). After 10 days, the cells were fixed with $3.7 \%$ methanol for $30 \mathrm{~min}$ at room temperature and stained with $0.1 \%$ crystal violet for $30 \mathrm{~min}$ at room temperature. Colonies consisting of $>50$ cells were counted manually under a light microscope (Olympus Corporation; magnification, $\mathrm{x} 40$ ).

Scratch-wound assay. Infected HaCaT and WS1 cells were seeded onto 6-well plates and cultured for $24 \mathrm{~h}$ at $37^{\circ} \mathrm{C}$ to form a $50 \%$ confluent monolayer. A straight scratch was gently and slowly created using a p200 pipette tip in the cell monolayer. Images of the wound were captured using a light microscope (Olympus Corporation; magnification, $\mathrm{x} 10$ ). Following two gentle washes with PBS, the plates were replenished with fresh DMEM containing 2\% FBS for regular culture. Images of the width of the scratched regions after 24 and $48 \mathrm{~h}$ were captured and wound closure was assessed using Image J Java 1.8.0-172 software (National Institute of Health). The relative wound width rate was calculated by dividing the changed distance in the scratched region by the initial distance.

Apoptosis assay. Early and late stage of apoptosis was evaluated using an Annexin V-FITC apoptosis detection kit (cat. no. C1062; Beyotime Institute of Biotechnology). HaCaT and WS1 cells were harvested $48 \mathrm{~h}$ post-infection and then stained with Annexin V/PI for $30 \mathrm{~min}$ at room temperature. To compute the percentage of apoptotic cells, a flow cytometer (FACSCalibur; BD Biosciences) with ModFit's LT v.3.0 software (BD Diagnostics) was used for data analysis.

Cell senescence assay. HaCaT and WS1 cells were fixed in $2 \%$ formaldehyde $/ 0.2 \%$ glutaraldehyde for $5 \mathrm{~min}$ at room temperature. $\beta$-Galactosidase staining solution containing X-gal (cat. no. C0602; Beyotime Institute of Biotechnology) was added after rinsing with PBS. The cells were then incubated for $6-10 \mathrm{~h}$ in a $37^{\circ} \mathrm{C}$ incubator without $\mathrm{CO}_{2}$. Senescent cells (stained blue) were observed and images were captured using light microscopy (Olympus Corporation; magnification, $\mathrm{x} 10$ ), and positive staining areas were calculated by determining the percentage of SA- $\beta$-gal ${ }^{+}$cells in five random fields in each of the three wells.

Cell cycle assay. HaCaT and WS1 cells were seeded in 100-mm petri dishes. At $24 \mathrm{~h}$, the cells were collected, washed with PBS and fixed with $70 \%$ precooled ethanol overnight at $4^{\circ} \mathrm{C}$. After the cells were stained with propidium iodide staining solution, including RNase A (50X; cat. no. C1052; Beyotime Institute of Biotechnology) in the dark for $30 \mathrm{~min}$ at $37^{\circ} \mathrm{C}$ to detect $S$ phase, $G_{1}$ phase and $G_{2} / M$ phase arrest. The cell cycle distribution was analyzed using a FACSCalibur system with ModFit's LT software.

RNA-Seq of gene expression and pathway-based analysis. Total RNA was extracted from infected WS1 cells with TRIzol ${ }^{\circledR}$ reagent (cat. no. 15596018; Invitrogen; Thermo Fisher Scientific, Inc.) and quantified by a NanoDrop ND-2000C spectrophotometer (Thermo Fisher Scientific, Inc.). RNA integrity was assessed using the Agilent 2100 Bioanalyzer (Agilent Technologies, Santa Clara, CA, USA). The libraries were subsequently constructed using a TruSeq Stranded mRNA LT Sample Prep kit (cat. no. RS-122-2302, Illumina, Inc.) according to the manufacturer's protocol. Transcriptome sequencing and analysis were conducted by Shanghai OE Biotech Co., Ltd. (Shanghai, China) http://oebiotech.bioon. com.cn/. The libraries were sequenced on an Illumina Novaseq platform (Illumina, Inc.) where 150 bp paired-end reads were generated. Raw data (raw reads) of fastq format were firstly processed using in-house perl scripts and the low-quality reads were removed to obtain the clean reads. The clean reads were subsequently mapped to the human genome (GRCh38) using Hierarchical Indexing for Spliced Alignment of Transcripts (Hisat2 v2.0.5.). Fragments per kilobase per million reads sequenced of each gene was calculated using StringTie, after which the read counts of each gene were obtained using featureCounts v1.5.0-p3. Differential expression analysis was performed using the DESeq2 $\mathrm{R}$ package (1.16.1). $\mathrm{P}<0.05$ and a fold-change $>2$ or $<0.5$ were set as the thresholds for significant differential expression. Hierarchical cluster analysis of differentially expressed genes (DEGs) was performed to demonstrate the expression pattern of genes in different groups and samples. Kyoto Encyclopedia of Genes and Genomes pathway enrichment analysis of DEGs were performed respectively using the clusterProfiler $\mathrm{R}$ package sed on the hypergeometric distribution. The Gene Expression Omnibus (GEO) number was GSE138621.

Statistical analysis. All the data are presented as the mean \pm SEM of at least three independent experiments and were analyzed using SPSS 19.0 software (SPSS Inc.). Differences between two groups were determined using a paired Student's t-test, and differences among $>2$ groups were analyzed by one-way analysis of variance and Tukey post hoc tests. $\mathrm{P}<0.05$ was considered to indicate a statistically significant difference.

\section{Results}

AUF1 promotes the viability and proliferation of skin cells. To explore the role of AUF1 in human skin cells, AUF1 was overexpressed or silenced using an adenovirus or a lentivirus, respectively. The efficiency of infection in $\mathrm{HaCaT}$ and WS1 cells was verified by RT-qPCR (Fig. 1A and B) and western blotting analysis (Fig. 1C and D). Compared with the sh-NC group, sh-AUF1-1- and sh-AUF1-2-infected cells demonstrated reduced levels of AUF1 protein. As expected, AUF1 protein expression in the Ad-AUF1-infected group was increased markedly As presented in Fig. 1A-D, AUF1 expression was successfully modulated by virus infection. Subsequently, cell viability was assayed using CCK-8-based analysis. As 
A

C
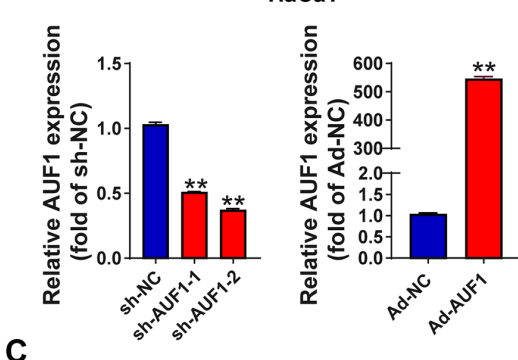

$\mathrm{HaCaT}$
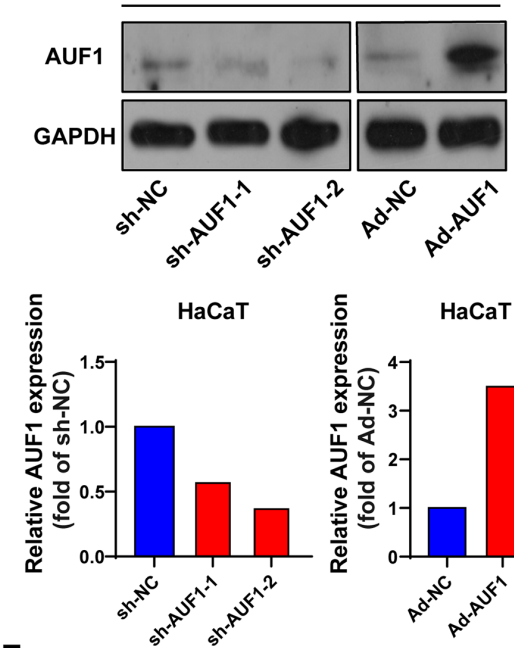

$\mathbf{E}$

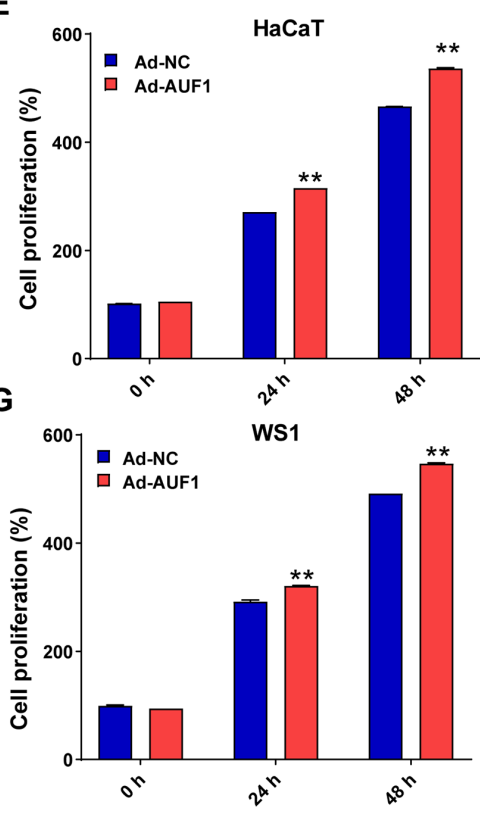

B

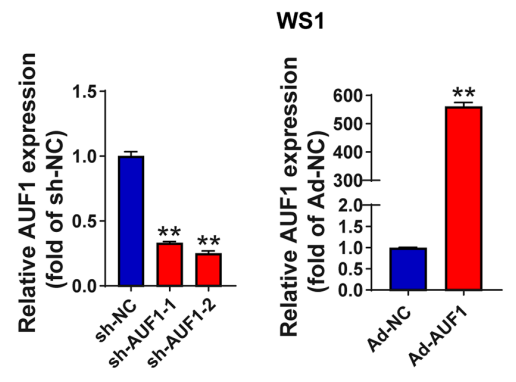

D

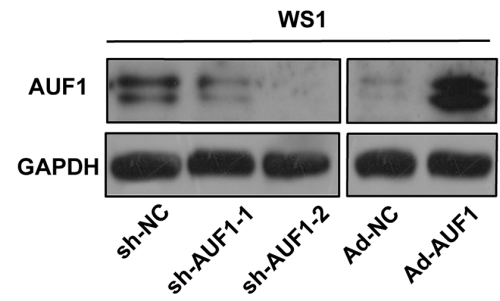

$\mathbf{F}$
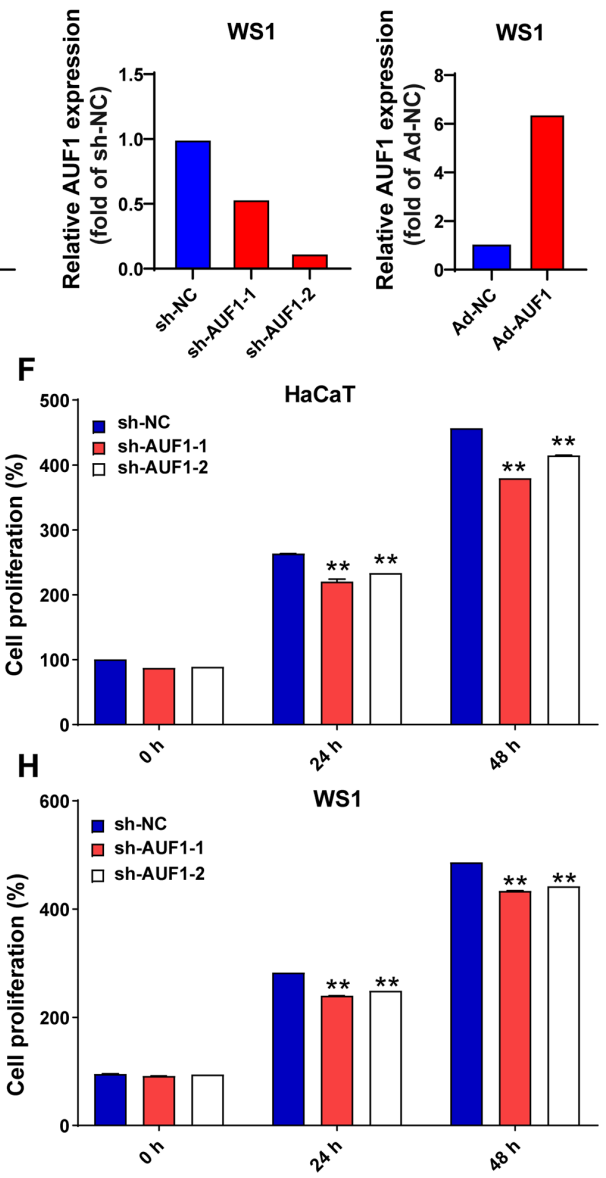

Figure 1. Knockdown and overexpression of AUF1 in human skin cells. (A) HaCaT cells and (B) WS1 cells were infected with AUF1-overexpressing adenovirus (Ad-AUF1) or AUF1-silencing lentiviruses (sh-AUF1/2) and their respective controls, and AUF1 mRNA levels were analyzed using reverse transcription-quantitative PCR. Western blotting analysis of AUF1 protein levels after infection with Ad-AUF1 or sh-AUF-1/2 and controls in (C) HaCaT and (D) WS1 cells. Cell proliferation of HaCaT cells following (E) AUF1 overexpression and (F) knockdown by Cell Counting Kit-8 analysis. Cell proliferation of WS1 cells following (G) AUF1 overexpression and (H) knockdown by Cell Counting Kit-8 analysis. ${ }^{* *} \mathrm{P}<0.01$ vs. Ad-NC or sh-NC. AUF1, AU-rich element RNA-binding factor 1; Ad, adenovirus; sh, short hairpin; NC, negative control.

presented in Fig. 1E-H, AUF1 overexpression significantly increased cell viability compared with Ad-NC at 24 and 48 h; whereas AUF1 silencing (sh-AUF1-1 and sh-AUF1-2) significantly suppressed cell viability in HaCaT and WS1 cells compared with sh-NC at 24 and $48 \mathrm{~h}$.

To explore the role of AUF1 in cell proliferation of skin cells, EdU staining and colony formation assays were performed. As presented in Fig. 2A and C, WS1 cells in the AUF1 overexpression group revealed a significantly increased EdU staining percentage compared with that in the control groups, while no significant difference was observed in $\mathrm{HaCaT}$ cells. Knockdown of AUF1 did not show a significant effect on the EdU-positive cell percentage in the two types of skin cells (Fig. 2B and D). 
A
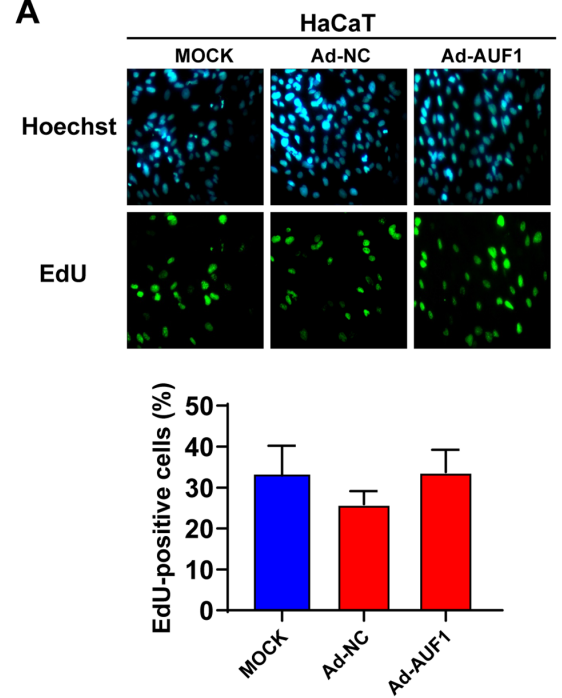

C
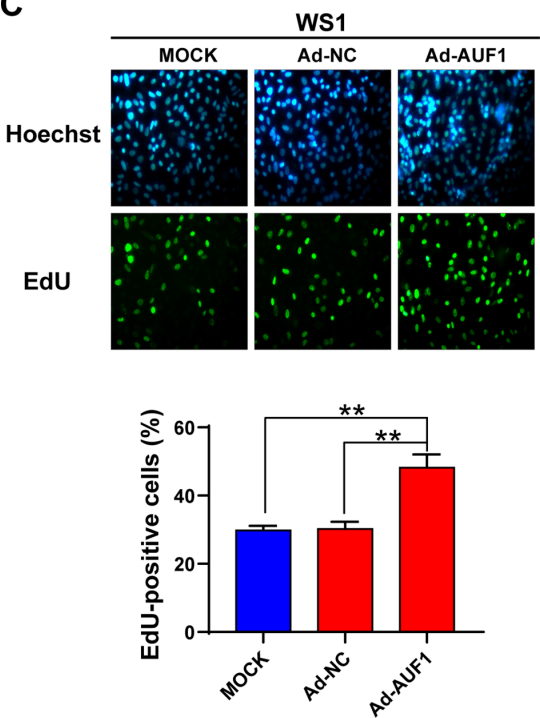

B
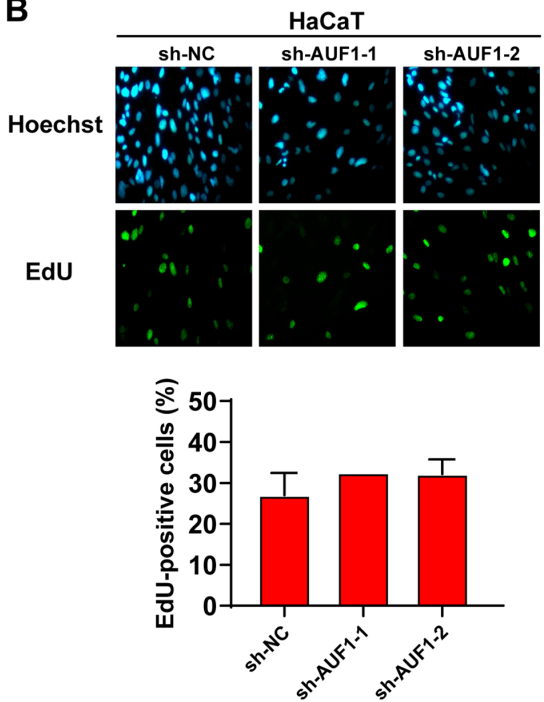

D
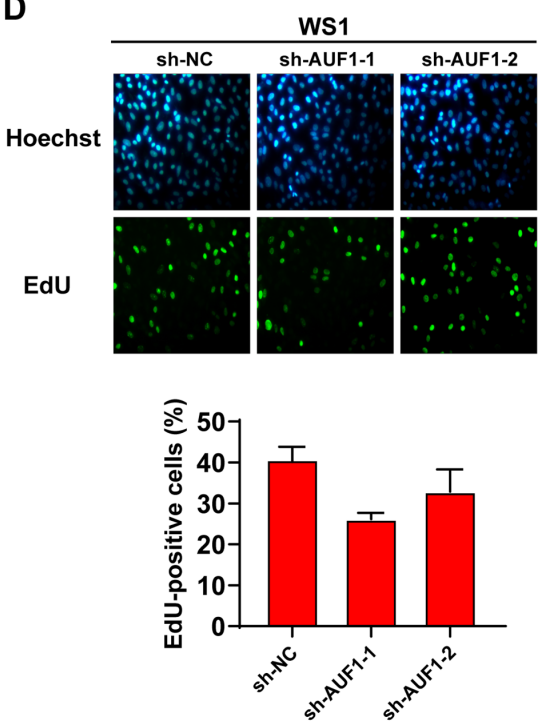

Figure 2. Effect of AUF1 on cell proliferation by EdU assay under the view of 20X light microscope objective lens. After virus infection, cell nuclei were stained with Hoechst 33342, showing blue fluorescence. EdU-labeled cells (green fluorescence) indicated new DNA synthesis. (A) HaCaT cells infected with Ad-AUF1 or Ad-NC. (B) HaCaT cells infected with sh-NC or AUF1-targeted lentiviruses (shRNA-1 and shRNA-2). (C) WS1 cells infected with Ad-AUF1 or Ad-NC. (D) Cells infected with sh-NC or AUF1-targeted lentiviruses (shRNA-1 and shRNA-2). ${ }^{* *} \mathrm{P}<0.01$. AUF1, AU-rich element RNA-binding factor 1; Ad, adenovirus; sh, short hairpin; NC, negative control.

Next, the role of AUF1 in skin cell colony formation was investigated. Overexpression of AUF1 resulted in a significant increase of $\mathrm{HaCaT}$ and WS1 colony foci compared with Ad-NC (Fig. 3A and C). Conversely, infection with the two AUF1-silencing lentiviruses caused a significant decrease in the number of colonies in both $\mathrm{HaCaT}$ cells and WS1 cells compared with sh-NC (Fig. 3B and D). Colony formation analysis consistently demonstrated that AUF1 expression promoted cell proliferation.

AUF1 enhances the migration of skin cells. A scratch-wound assay was used to evaluate cell migration, which is important for wound healing. As presented in Fig. 4A and C, when compared with mock or Ad-NC group, the migration of HaCaT and WS1 cells was significantly increased with AUF1 overexpression at 24 and $48 \mathrm{~h}$. By contrast, when compared with the sh-NC group, fewer AUF1-silenced HaCaT and WS1 cells migrated toward the center of the gap of the cells, leading to a decreased rate of wound healing at 24 and $48 \mathrm{~h}$ (Fig. 4B and D). Migration inhibition induced by AUF1 depletion was observed even after incubation for $48 \mathrm{~h}$. Overall, these results demonstrated that AUF1 expression can affect the migration of skin cells.

AUF1 attenuates cell senescence in skin cells. Cell senescence was analyzed using $\beta$-galactosidase staining, and the relative numbers of $\beta$-galactosidase-positive skin cells were identified. In the AUF1 overexpression group of skin cells, there was a significant decrease in the number of $\beta$-galactosidase-positive cells following infection with AUF1-overexpressing adenovirus compared with the Ad-NC groups (Fig. 3E and G). The positive areas were significantly reduced by Ad-AUF1 infection in $\mathrm{HaCaT}$ and WS1 cells (Fig. 3E and G). Conversely, as presented in Fig. 3F and $\mathrm{H}$, knocking down AUF1 nearly tripled the $\beta$-galactosidase staining-positive areas in the two shRNA lentivirus-infected HaCaT cells and WS1 cells 
A
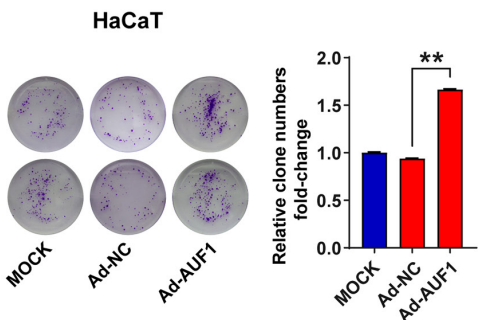

C

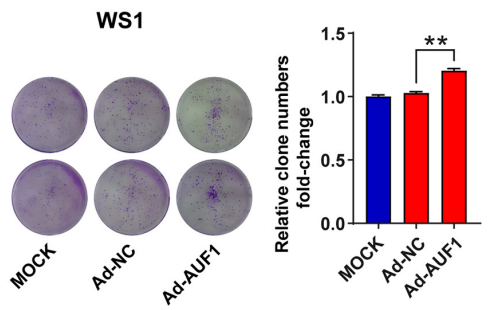

E

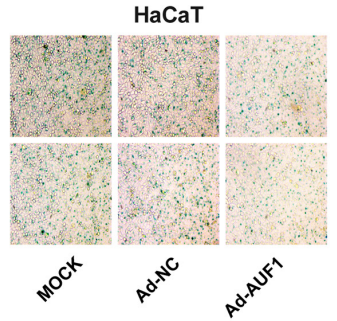

G

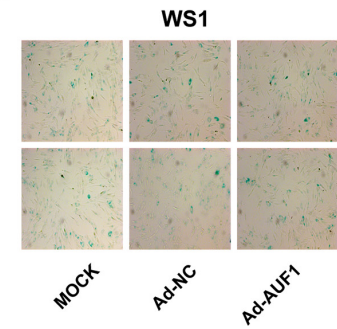

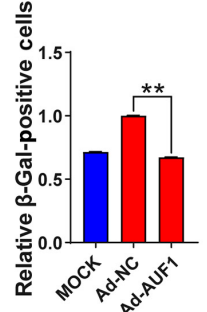

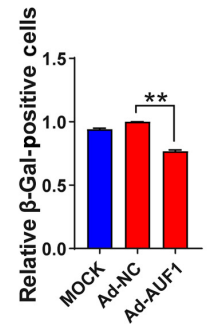

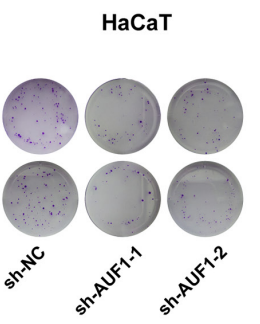

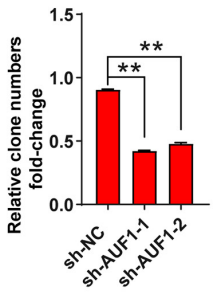

D
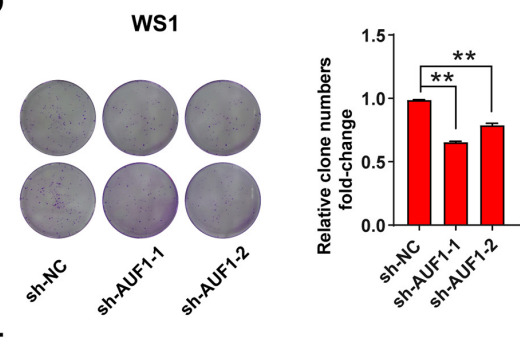

$\mathbf{F}$
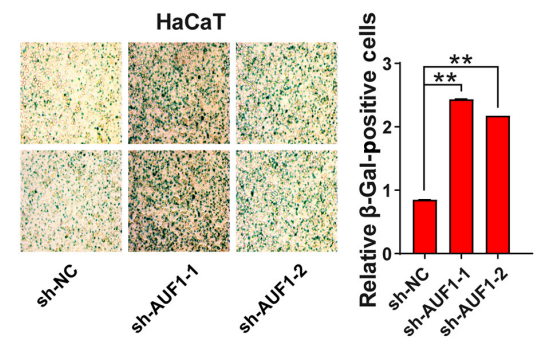

H

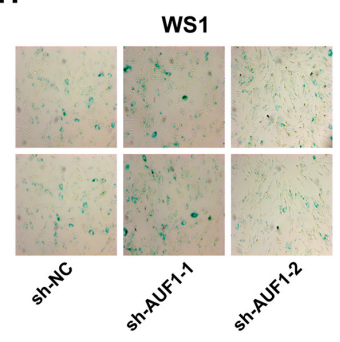

Figure 3. Effect of AUF1 on proliferation by colony formation assay and senescence in skin cells. Cells were seeded at a density of 1,000 cells per dish. After 10 days, cells were stained with crystal violet. Colonies with $>50$ cells were counted. HaCaT cells infected with (A) Ad-AUF1 or Ad-NC or (B) sh-NC or AUF1-targeted lentiviruses (shRNA-1 and shRNA-2). WS1 cells infected with (C) Ad-AUF1 or Ad-NC or (D) with sh-NC or AUF1-targeted lentiviruses (shRNA-1 and shRNA-2). Senescence of HaCaT cells following (E) AUF1 overexpression and (F) knockdown. Senescence of WS1 cells following (G) AUF1 overexpression and $(\mathrm{H})$ knockdown. $\beta$-Galactosidase staining was used to measure cell senescence. The left panel shows representative images of stained senescent cells. ${ }^{* *} \mathrm{P}<0.01$. AUF1, AU-rich element RNA-binding factor 1; Ad, adenovirus; sh, short hairpin; NC, negative control.

compared with the sh-NC. These results reflected the positive function of AUF1 in cell senescence.

AUF1 decreases apoptosis in skin cells. As cell proliferation was affected by AUF1 expression, the function of AUF1 expression on apoptosis was investigated using an Annexin V/PI double-staining assay. Due to the pretreatment time before cytometry analysis, there were higher basal levels of apoptosis in HaCaT cells. The Ad-AUF1 groups in both $\mathrm{HaCaT}$ and WS1 cells demonstrated significantly reduced apoptosis rates compared with the control groups; by contrast, the apoptosis rates of AUF1-downregulated skin cells were significantly increased compared with the controls (Fig. 5A-F). These results indicated the antiapoptotic role of AUF1 in skin cells.

AUF1 has no significant effect on cell cycle progression. AUF1 may play a role in cell proliferation by disturbing the cell cycle; therefore, a cell cycle analysis was conducted using flow cytometry. However, no statistical significance for the percentage of cells in different phases was observed between any infected group (either the AUF1 overexpression group or AUF1 knockdown groups) and the corresponding control group in the two types of cells (Fig. 5G-J). As presented in Fig. $5 \mathrm{~K}-\mathrm{L}, \mathrm{AUF} 1$ did not modulate cell cycle progression in $\mathrm{HaCaT}$ and WS1 cells.

Transcripts and pathways that are affected by AUF1. As AUF1 is an RBP, multiple transcripts and pathways may be affected by the action of AUF1. To explore AUF1-affected mRNAs, WS1 cells with AUF1 overexpression and silencing were used to perform RNA-Seq and KEGG pathway analysis (Fig. 6A). The raw data are accessible at GSE138621. The results indicated that knockdown of AUF1 influenced >10,000 mRNAs, while overexpression of AUF1 caused a significant expression 
A
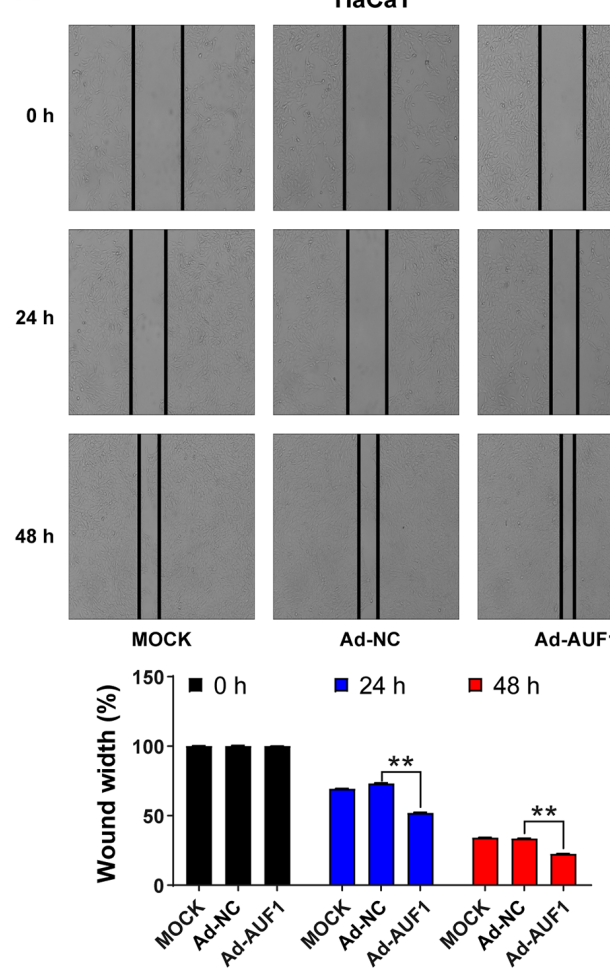

C
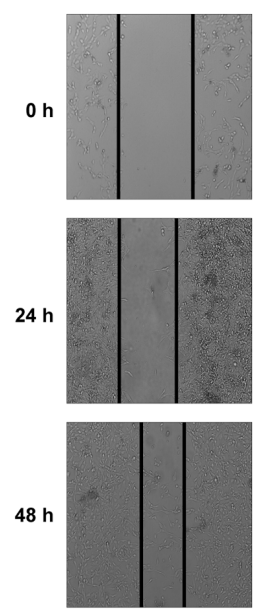

MOCK
HaCaT
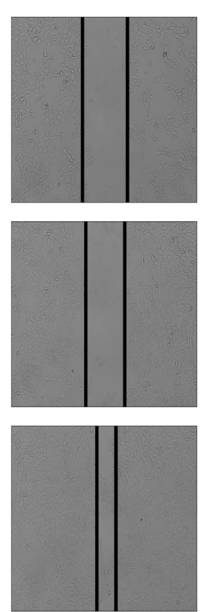

Ad-NC

WS1
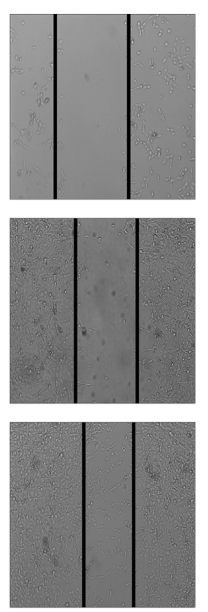

Ad-NC
Ad-AUF1

Ad-AUF1

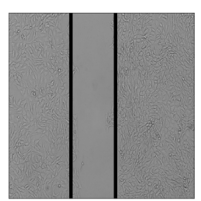

B
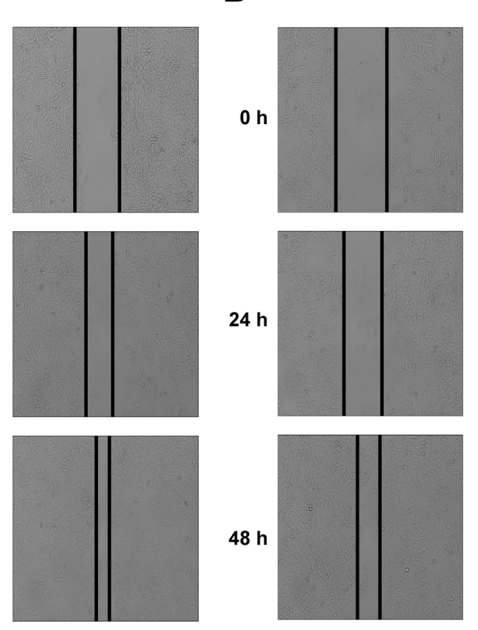

sh-NC

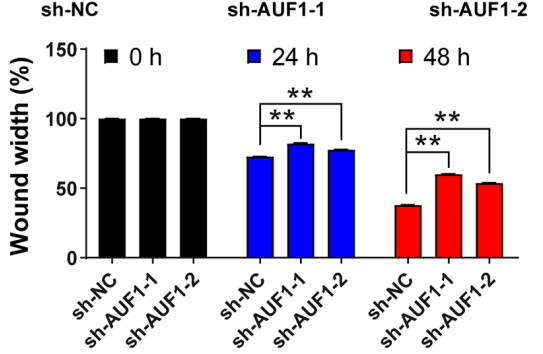

D
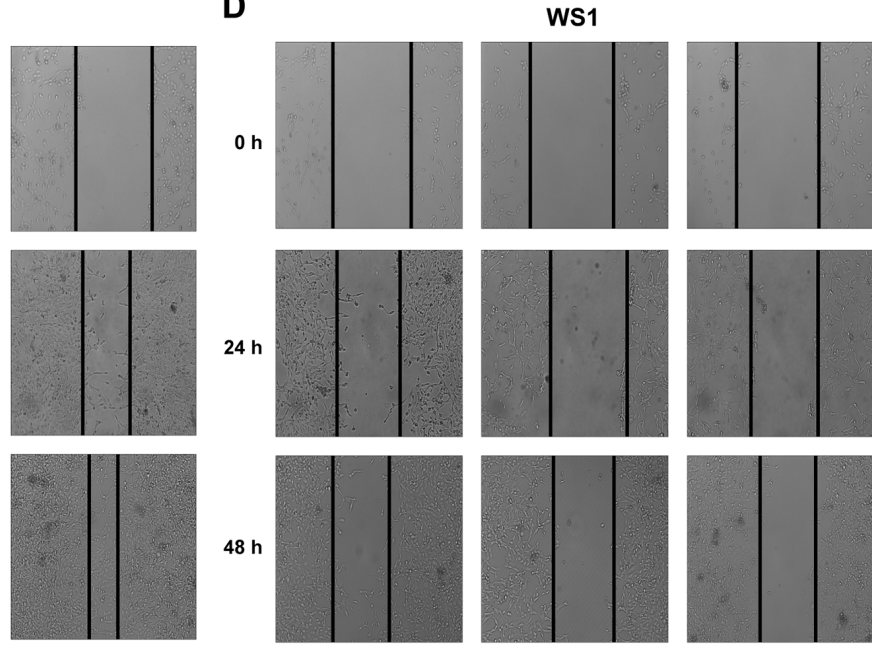

sh-NC

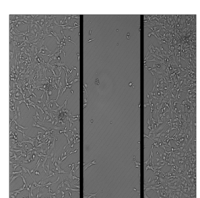

sh-AUF1-1
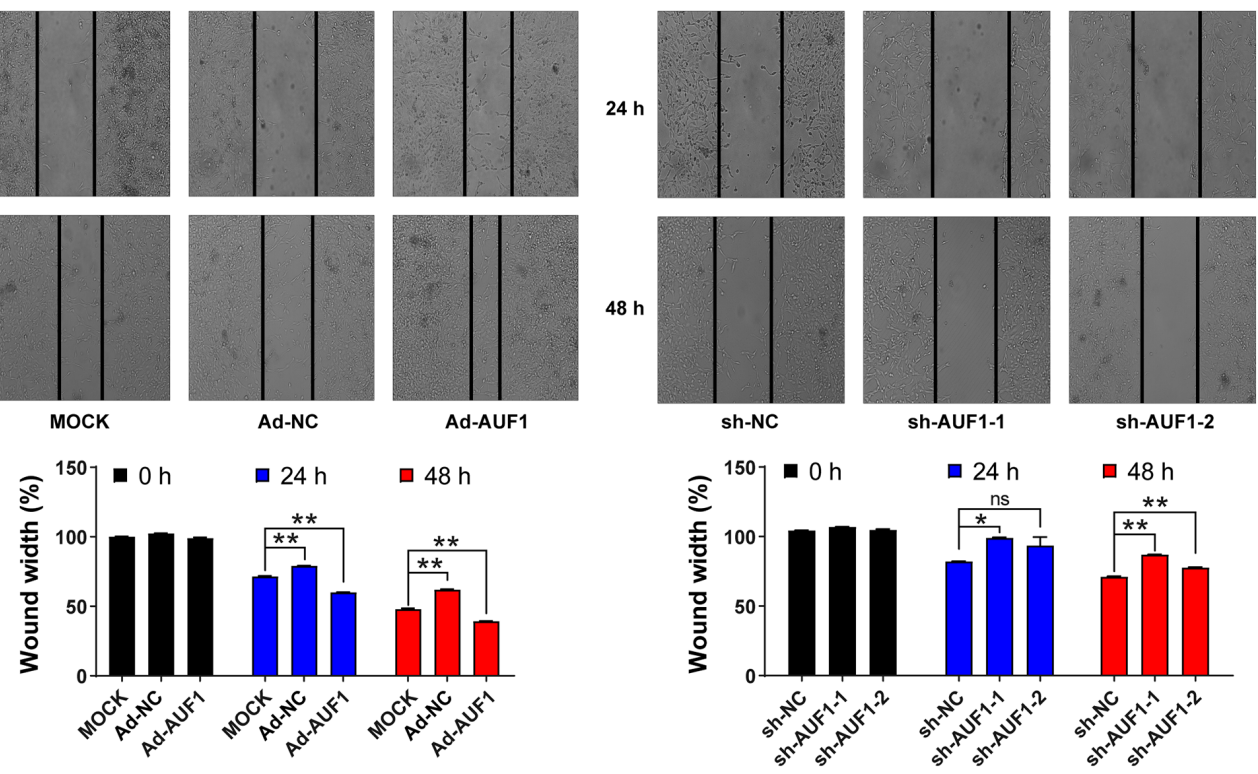

Figure 4. Effect of AUF1 on skin cell migration. HaCaT or WS1 cell migration after infection with the indicated viruses. Images of the scratched region were captured at 0,24 and $48 \mathrm{~h}$ after the wounds were created, and the relative wound width rate was calculated by dividing the distance of the scratched region by the initial distance. HaCaT cells infected with (A) Ad-AUF1, Ad-NC or the mock, or (B) sh-NC or AUF1-targeted lentiviruses (shRNA-1 and shRNA-2). WS1 cells infected with (C) Ad-AUF1, Ad-NC or the mock, or (D) with sh-NC or AUF1-targeted lentiviruses (shRNA-1 and shRNA-2). ${ }^{*}<0.05$, ${ }^{* *} \mathrm{P}<0.01$. AUF1, AU-rich element RNA-binding factor 1; sh, short hairpin; NC, negative control; ns, not significant.

change in 6,771 mRNAs (Fig. 6B-D). A total of 6,771 mRNAs differentially expressed in AUF1-overexpressing cells included 3,422 up- and 3,349 downregulated mRNAs. AUF1 silencing involved 5,966 up- and 6,023 downregulated transcripts (Fig. 6D). A total of 18 mRNAs (eight mRNAs with positive associations and $10 \mathrm{mRNAs}$ with negative associa- 
A

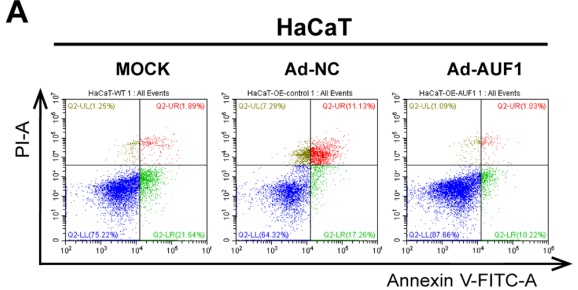

c

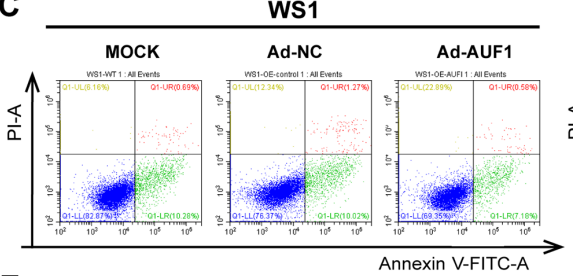

E

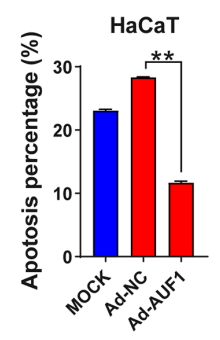

G

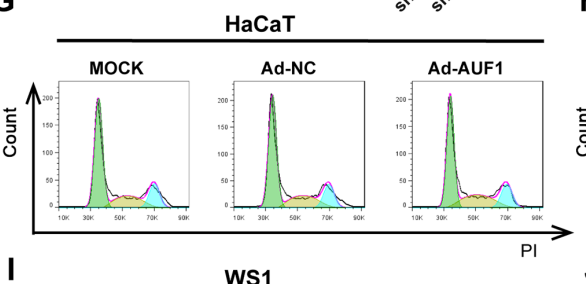

B
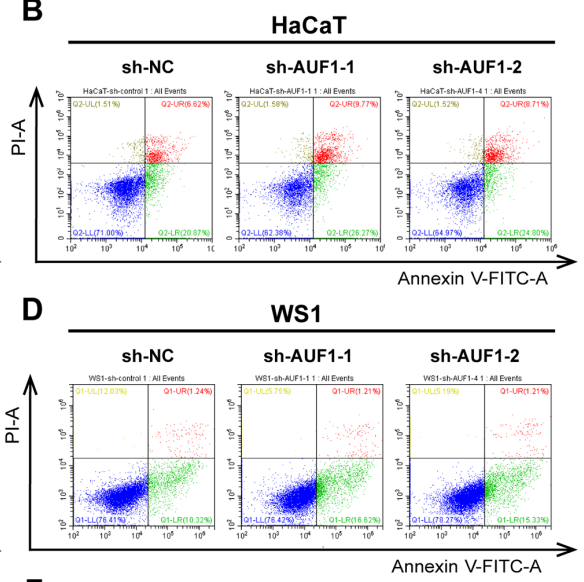

$F$

F ws

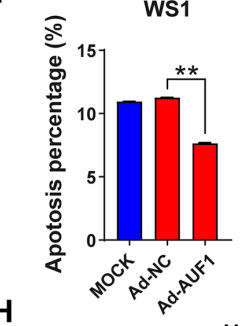

HaCaT
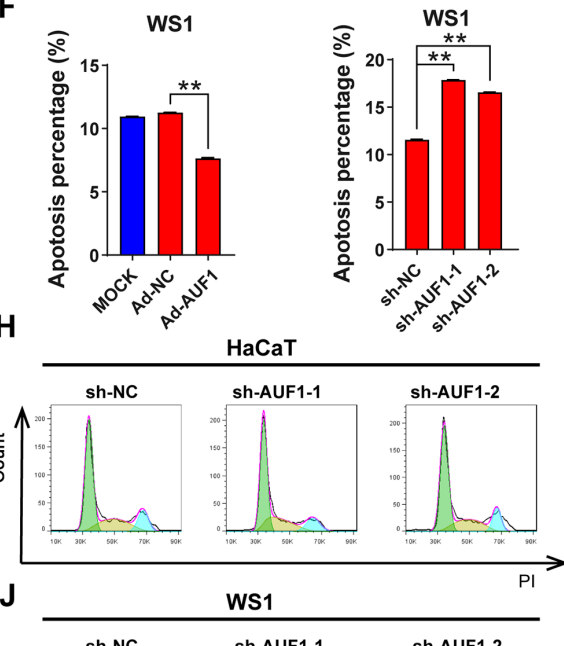

sh-AUF1-1 sh-AUF1-2

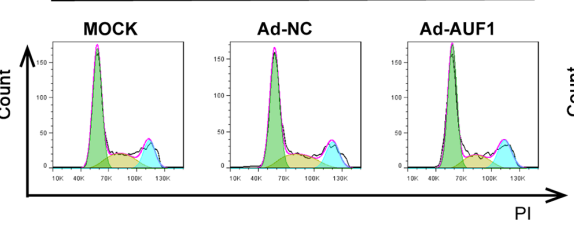

K

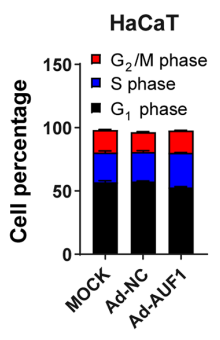

HaCaT

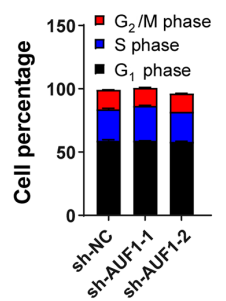

L
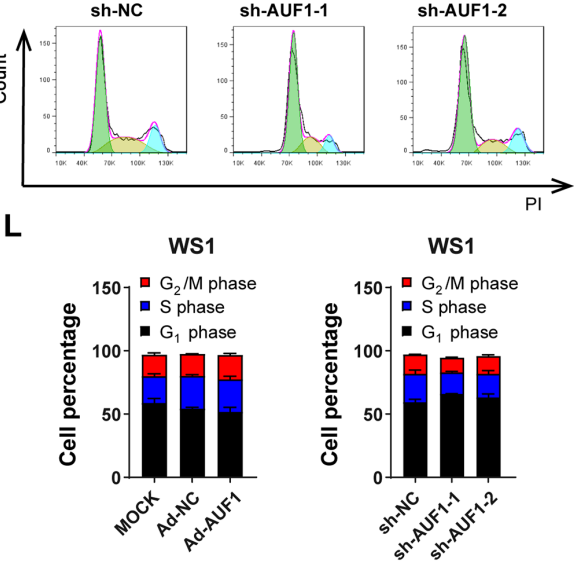

Figure 5. Effect of AUF1 on apoptosis and the cell cycle of skin cells. Apoptosis of HaCaT cells with (A) AUF1 overexpression and (B) knockdown. The cells were infected with the indicated viruses. After $48 \mathrm{~h}$, the apoptosis rate was measured using Annexin V/PI double staining. Apoptosis of WS1 cells with (C) AUF1 overexpression or (D) knockdown. Quantification of (E) HaCaT and (F) WS1 apoptosis rates. Cell cycle distributions of HaCaT cells with (G) AUF1 overexpression or (H) knockdown, and WS1 cells with (I) AUF1 overexpression or (J) knockdown. Quantification of (K) HaCaT and (L) WS1 cell cycle distributions. ${ }^{* *} \mathrm{P}<0.01$. AUF1, AU-rich element RNA-binding factor 1; Ad, adenovirus; sh, short hairpin; NC, negative control; ns, not significant.

tions) demonstrated consistent associations with both AUF1 overexpression and silencing (Fig. 6D): Mitochondrially encoded cytochrome $\mathrm{B}$, anti-muellerian hormone (AMH), secreted protein acidic and cysteine rich (SPARC), forkhead box G1 (FOXG1), G protein-coupled receptor class c group 5 member A, cysteine rich angiogenic inducer 61 (CYR61), NADH-ubiquinone oxidoreductase chain 3, C-X-C motif chemokine ligand 6 , oligosaccharyltransferase complex subunit 4, non-catalytic, mitochondrially encoded ATP synthase membrane subunit 6 , phosphoserine aminotrans- ferase 1, solute carrier family 7 member 5, methionyl-TRNA synthetase 1 (MARS), glycyl-TRNA synthetase 1, phospholipid scramblase 1 (PLSCR1), 2'-5'-oligoadenylate synthetase 1, nuclear autoantigen Sp-100 (SP100), chromosome 17 open reading frame 51 (Fig. 6E). KEGG pathway analysis revealed AUF1-affected networks involved multiple biological processes, including cell adhesion molecules, the proteasome and the spliceosome (Fig. 7A and B). mRNAs downregulated by AUF1 were associated with cell adhesion molecules, pathways in cancer, cellular senescence and the TGF- $\beta$ signaling 
A

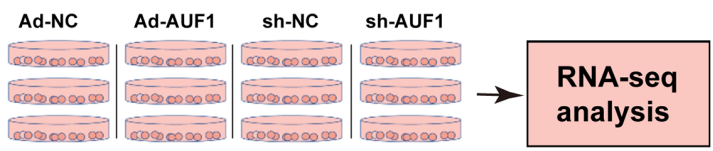

B

C
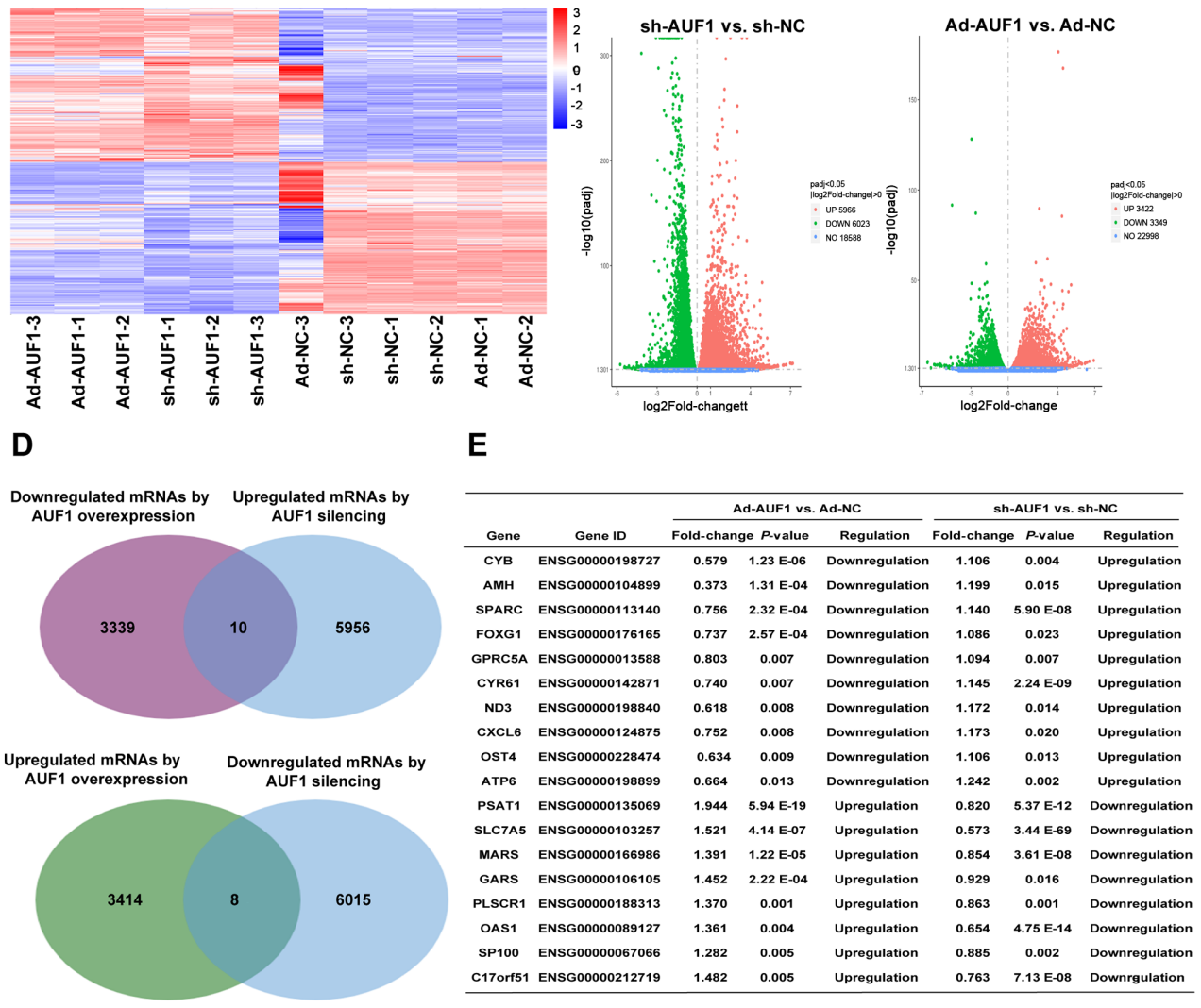

Figure 6. RNA-Seq analysis of the transcriptome with AUF1 overexpression and knockdown in WS1 cells. (A) Experimental design of the AUF1 RNA-Seq analysis. A total of four groups of WS1 cells were used, including Ad-NC, Ad-AUF1, sh-NC and sh-AUF1 cells (n=3). (B) Heatmap of mRNA expression incorporating all the sequencing samples. (C) Volcano plots showing the differential expression of transcripts between AUF1 overexpression and control conditions (Ad-AUF1 vs. Ad-NC) and between AUF1 downregulation and control shRNA conditions (sh-AUF1 vs. sh-NC). The green dots represent the downregulated transcripts, and the red dots denote the upregulated transcripts. (D) Venn diagram for overlapping analysis of differentially expressed transcripts. (E) Overall, there are 18 common dysregulated mRNAs in the Ad-AUF1 vs. Ad-NC and sh-AUF1 vs. sh-NC groups. RNA-Seq, RNA-sequencing; Ad, adenovirus; sh, short hairpin; NC, negative control.

pathway, among others (Fig. 7C and D). These results suggested a complex regulatory network of AUF1 in skin cells.

\section{Discussion}

AUF1 regulates various cell processes, such as cell proliferation, apoptosis, cell cycle progression and/or senescence, by interacting with adenylate-uridylate (AU)-rich element-bearing mRNAs $(7,11,16,17)$. The functional significance of AUF1 has been explored in various types of cells, including tumor cells and noncancerous cells $(7-11,16-18)$. However, to the best of our knowledge, the role of AUF1 in skin cells has not been reported, even in skin diseases.

The present study aimed to explore whether AUF1 played a notable role in the homeostasis of skin cells in vitro. It was revealed that, when WS1 cells were infected with different types of viruses, there were expected changes in AUF1 expression. These included AUF1 downregulation when exposed to AUF1 silencing viruses and AUF1 overexpression following
Ad-AUF1 infection. Generally, overexpression of AUF1 promoted the proliferation and migration of $\mathrm{HaCaT}$ and WS1 cells, while knockdown of AUF1 demonstrated the opposite effects. Although $\mathrm{HaCaT}$ and WS1 cells are non-cancerous cells, they are immortalized by the American Type Culture Collection. A previous report indicated that $\mathrm{HaCaT}$ cells have various capacities for cloning (19). The present study used colony formation assays to confirm the pro-proliferative role of AUF1, as AUF1 has been reported to stimulate cell proliferation in human chronic myeloid leukemia K562 cells (20). Consistently, downregulation of AUF1 expression has been reported to reduce cell proliferation in ESCC cells and human colorectal carcinoma HCT116 cells $(7,16)$. However, a limitation of the present study was that an in vivo study based on animal models was not performed. In vitro results may be different to those of cells in vivo and primary skin cells. When AUF1 is continuously overexpressed, the cells may be regulated by other mechanisms to control cell proliferation as AUF1 is constitutively expressed in normal skin tissues (21). 
A

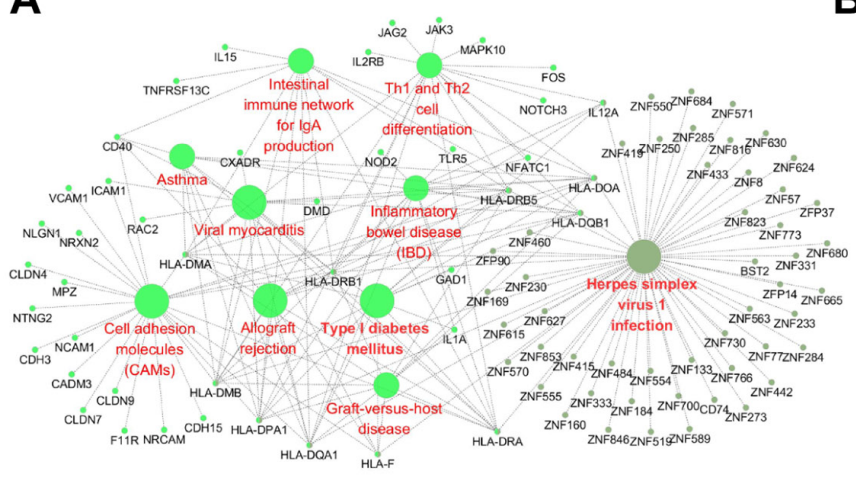

B

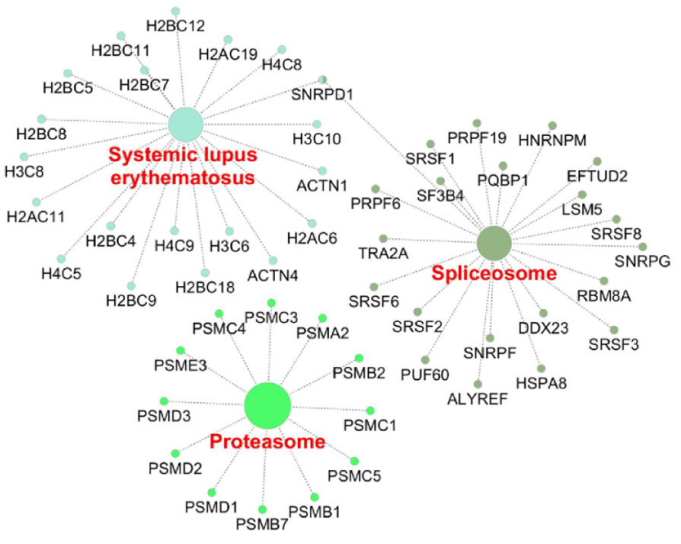

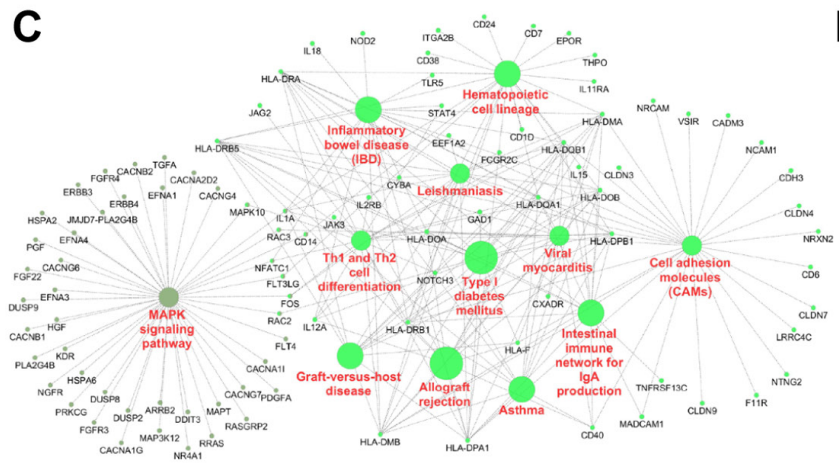

D

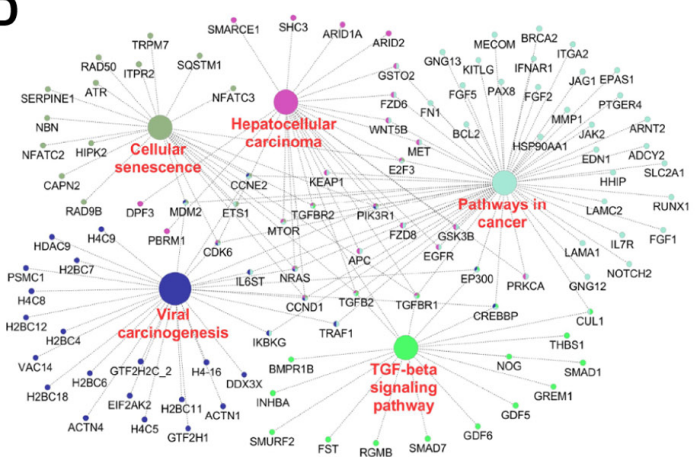

Figure 7. KEGG pathway analysis of dysregulated mRNAs. KEGG pathway analysis of (A) upregulated and (B) downregulated mRNAs after AUF1 overexpression. KEGG pathway analysis of (C) upregulated and (D) downregulated mRNAs after AUF1 downregulation. KEGG, Kyoto Encyclopedia of Genes and Genomes.

However, the difference between in vitro and in vivo conditions warrants further investigation. In vivo experiments will be considered for improved evaluation of the role of AUF1 in skin cells in follow-up studies. Regarding the effect on cell migration, AUF1 served as a promoter in the present study, which was consistent with a previous study which demonstrated that AUF1 expression and wound healing were associated (18). Similarly, AUF1 overexpression contributes to cell migration (22). However, AUF1 knockdown in B16 mouse melanoma cells is beneficial to cell migration (23).

The present study them examined whether AUF1 was associated with apoptosis, senescence and cell cycle alteration. Apoptosis was significantly decreased with AUF1 overexpression, but was enhanced by AUF1 knockdown in HaCaT and WS1 cells. Cell senescence was affected in a similar pattern as apoptosis, whereas cell cycle changes were not significant after the cells were treated with the different viruses. Apoptosis and senescence reflected the cell proliferation ability and cell motility modulated by AUF1, which is consistent with observations in a report involving ESCC cells (7). This indicates that silencing AUF1 inhibits the proliferation and enhances the apoptosis of ESCC cells (7). AUF1 has been suggested to have an inhibitory effect on apoptosis, and upregulated AUF1 contributes to uncontrolled proliferation of liver cells (10). Previous studies have demonstrated that AUF1 suppresses cellular senescence, and plays roles in anti-aging and maintaining cell proliferation $(11,24)$. However, the cell cycle was not affected by AUF1 in the present study, although
AUF1 has been considered to control the mRNA decay of cell cycle-regulatory proteins $(6,25)$. It was hypothesized that the pro-proliferative role of AUF1 was likely attributed to decreased apoptosis and senescence. In addition, a number of examples demonstrate that cell proliferation is associated with cell death, meaning that changes of the cell cycle are not essential (26-30). Thus, a more sophisticated mechanism other compared with the cell cycle was implicated in AUF1 modulation of skin cells.

Diverse targets of AUF1 have been explored to elucidate the mechanism of AUF1 in different cell types, such as GTP cyclohydrolase 1 for cell proliferation in ESCC cells, neural precursor cell expressed developmentally downregulated 4-like in HK2 cells and myocyte-specific enhancer factor $2 \mathrm{C}$ for the myogenesis program in mouse myoblasts $(1,18,26,31)$. To explore the underlying mechanism of AUF1 in skin cells, the present study performed RNA-Seq, which revealed the landscape of the transcriptome in skin cells with up/downregulated AUF1 expression. Thousands of genes demonstrated differential expression following AUF1 upregulation or downregulation, and hundreds of pathways were subsequently annotated with KEGG pathway functions. By integrating RNA-Seq data from skin cells with AUF1 upregulation and downregulation, 18 mRNAs (eight mRNAs with positive associations and 10 mRNAs with negative associations) revealed consistent associations with AUF1 expression levels. Among these 18 potentially regulated AUF1 molecules, several have been demonstrated to be associated with cell function. For example, 
PAST homolog 1 contributes to cell proliferation and cell cycle progression $(32,33)$. Reduced expression of 2-5A synthase 1 by a non-coding RNA named TINCR facilitates proliferation in breast cancer cells (34). MARS has an important role in initiating translation and protection against cellular damage (35). MARS has been revealed to promote the proliferation of cancer cells (36). Taken together, AUF1 exerted its influence through wide-spectrum targets, which was consistent with its behavior in other types of cell, such as ESCC cells, human colorectal carcinoma HCT116 cells and human chronic myeloid leukemia K562 cells $(7,16,21)$. However, the targets of AUF1 are likely to be cell-type specific. Querying published papers revealed a number of genes that could be the exact targets for AUF1 that lead to cell proliferation, including AMH, SPARC, FOXG1, CYR61, PLSCR1 and SP100 (37-42). These genes were explored by RNA-Seq in the present study. Among them, CYR61 (CCN1) and SP100 are associated with apoptosis, and FOXG1 is connected with cell senescence (43-45). However, which one could be the exact target for AUF1 that leads to cell proliferation needs to be further studied.

In conclusion, AUF1 played a positive role in the proliferation and migration of immortalized skin cells. AUF1 inhibited cell senescence and apoptosis in human skin cells and modulated downstream mRNAs implicated in multiple pathways in skin cells.

\section{Acknowledgements}

Not applicable.

\section{Funding}

This work is supported by the National Natural Science Foundation of China (grant nos. 31770911, 81803166, 32071238 and 82073477), Military Logistics Research Program (grant no. BKJ18J003), the Young Talent Project of China National Nuclear Corporation and the Fundamental Research Funds for the Central Universities.

\section{Availability of data and materials}

The datasets generated and/or analyzed during the current study are available in the Gene Expression Omnibus repository, https://www.ncbi.nlm.nih.gov/geo/query/acc. cgi?acc=GSE138621.

\section{Authors' contributions}

DY and XL conceived the experiments and designed the research. DY, XL and ZW performed the molecular biology experiments. SJ, TY and ZJ analyzed and interpreted the data. SZ, YS and KF performed statistical analysis. SZ drafted the manuscript. KF performed language editing of the manuscript. YS provided general supervision. ZJ and KF confirm the authenticity of all the raw data. All authors have read and approved the final manuscript.

\section{Ethics approval and consent to participate}

Not applicable.

\section{Patient consent for publication}

Not applicable.

\section{Competing interests}

The authors declare that they have no competing interests.

\section{References}

1. Stavast CJ and Erkeland SJ: The Non-Canonical Aspects of MicroRNAs: Many Roads to Gene Regulation. Cells 8: 1465, 2019.

2. Loflin $P$, Chen CY and Shyu AB: Unraveling a cytoplasmic role for hnRNP D in the in vivo mRNA destabilization directed by the AU-rich element. Genes Dev 13: 1884-1897, 1999.

3. Zhang W, Wagner BJ, Ehrenman K, Schaefer AW, DeMaria CT, Crater D, DeHaven K, Long L and Brewer G: Purification, characterization, and CDNA cloning of an AU-rich element RNA-binding protein, AUF1. Mol Cell Biol 13: 7652-7665, 1993.

4. Wagner BJ, DeMaria CT, Sun Y, Wilson GM and Brewer G: Structure and genomic organization of the human AUF1 gene: Alternative pre-mRNA splicing generates four protein isoforms. Genomics 48: 195-202, 1998.

5. Zucconi BE and Wilson GM: Modulation of neoplastic gene regulatory pathways by the RNA-binding factor AUF1. Front Biosci (Landmark Ed) 16: 2307-2325, 2011.

6. Lozano-Rosas MG, Chávez E, Velasco-Loyden G, Domínguez-López M, Martínez-Pérez L and Chagoya De Sánchez V: Diminished S-adenosylmethionine biosynthesis and its metabolism in a model of hepatocellular carcinoma is recuperated by an adenosine derivative. Cancer Biol Ther 21: 81-94, 2020.

7. Gao Y, Wang W, Cao J, Wang F, Geng Y, Cao J, Xu X, Zhou J, Liu P and Zhang S: Upregulation of AUF1 is involved in the proliferation of esophageal squamous cell carcinoma through GCH1. Int J Oncol 49: 2001-2010, 2016.

8. Sarkar S, Sinsimer KS, Foster RL, Brewer G and Pestka S: AUF1 isoform-specific regulation of anti-inflammatory IL10 expression in monocytes. J Interferon Cytokine Res 28: 679-691, 2008.

9. Trojanowicz B, Sekulla C, Dralle H and Hoang-Vu C: Expression of ARE-binding proteins AUF1 and HuR in follicular adenoma and carcinoma of thyroid gland. Neoplasma 63: 371-377, 2016.

10. Wu X, Yang Y, Huang Y, Chen Y, Wang T, Wu S, Tong L, Wang Y, Lin L, Hao M, et al: RNA-binding protein AUF1 suppresses miR-122 biogenesis by down-regulating Dicer1 in hepatocellular carcinoma. Oncotarget 9: 14815-14827, 2018.

11. He J, Jiang YF, Liang L, Wang DJ, Wei WX, Ji PP, Huang YC, Song H, Lu XL and Zhao YX: Targeting of AUF1 to vascular endothelial cells as a novel anti-aging therapy. J Geriatr Cardiol 14: 515-523, 2017.

12. Zhang S, Xue J, Zheng J, Wang S, Zhou J, Jiao Y, Geng Y, Wu J, Hannafon BN and Ding WQ: The superoxide dismutase 13 'UTR maintains high expression of the SOD1 gene in cancer cells: The involvement of the RNA-binding protein AUF-1. Free Radic Biol Med 85: 33-44, 2015.

13. Gund R, Zirmire R, J H, Kansagara G and Jamora C: Histological and Immunohistochemical Examination of Stem Cell Proliferation and Reepithelialization in the Wounded Skin. Bio Protoc 11: e3894, 2021.

14. Sadri N and Schneider RJ: Auf1/Hnrnpd-deficient mice develop pruritic inflammatory skin disease. J Invest Dermatol 129: 657-670, 2009.

15. Livak KJ and Schmittgen TD: Analysis of relative gene expression data using real-time quantitative PCR and the 2(-Delta Delta C(T)) method. Methods 25: 402-408, 2001.

16. Ma W, Qiao J, Zhou J, Gu L and Deng D: Characterization of novel LncRNA P14AS as a protector of ANRIL through AUF1 binding in human cells. Mol Cancer 19: 42, 2020.

17. Yan J, Du F, Li SD, Yuan Y, Jiang JY, Li S, Li XY and Du ZX: AUF1 modulates TGF- $\beta$ signal in renal tubular epithelial cells via post-transcriptional regulation of Nedd4L expression. Biochim Biophys Acta Mol Cell Res 1865: 48-56, 2018.

18. Al-Khalaf HH and Aboussekhra A: AUF1 positively controls angiogenesis through mRNA stabilization-dependent up-regulation of HIF- $1 \alpha$ and VEGF-A in human osteosarcoma. Oncotarget 10: 4868-4879, 2019. 
19. Tate S, Imai M, Matsushita N, Nishimura EK, Higashiyama S and Nanba D: Rotation is the primary motion of paired human epidermal keratinocytes. J Dermatol Sci 79: 194-202, 2015.

20. Lu M, Pan C, Zhang L, Ding C, Chen F, Wang Q, Wang K and Zhang X: ING4 inhibits the translation of proto-oncogene MYC by interacting with AUF1. FEBS Lett 587: 1597-1604, 2013.

21. Moore AE, Chenette DM, Larkin LC and Schneider RJ: Physiological networks and disease functions of RNA-binding protein AUF1. Wiley Interdiscip Rev RNA 5: 549-564, 2014.

22. Qian W, Cai X, Qian Q, Wang D and Zhang L: Angelica sinensis polysaccharide suppresses epithelial-mesenchymal transition and pulmonary fibrosis via a DANCR/AUF-1/FOXO3 regulatory axis. Aging Dis 11: 17-30, 2020.

23. Sun S, Zhang X, Lyu L, Li X, Yao S and Zhang J: Autotaxin expression is regulated at the post-transcriptional level by the RNA-binding proteins HuR and AUF1. J Biol Chem 291: 25823-25836, 2016.

24. Lee JW, Chun YL, Kim AY, Lloyd LT, Ko S, Yoon JH and Min KW: Accumulation of mitochondrial RPPHI RNA is associated with cellular senescence. Int J Mol Sci Jan 22: 782, 2021.

25. Panda AC, Abdelmohsen K, Yoon JH, Martindale JL, Yang X, Curtis J, Mercken EM, Chenette DM,Zhang Y, Schneider RJ, et al: RNA-binding protein AUF1 promotes myogenesis by regulating MEF2C expression levels. Mol Cell Biol 34: 3106-3119, 2014

26. Xu Y, Jiang Y, Wang Y, Ren Y, Zhao Z, Wang T and Li T: LINC00473 regulated apoptosis, proliferation and migration but could not reverse cell cycle arrest of human bone marrow mesenchymal stem cells induced by a high-dosage of dexamethasone. Stem Cell Res (Amst) 48: 101954, 2020.

27. Alimirah F, Pulido T, Valdovinos A, Alptekin S, Chang E, Jones E, Diaz DA, Flores J, Velarde MC, Demaria M, et al: Cellular senescence promotes skin carcinogenesis through p38MAPK and p44/42MAPK signaling. Cancer Res 80 3606-3619, 2020.

28. Walczak K, Langner E, Makuch-Kocka A, Szelest M, Szalast K, Marciniak S, Plech T: Effect of Tryptophan-Derived AhR Ligands, Kynurenine, Kynurenic Acid and FICZ, on Proliferation, Cell Cycle Regulation and Cell Death of Melanoma Cells-In Vitro Studies. Int J Mol Sci. 21:7946, 2020.

29. Lin Q, Jin HJ, Zhang D and Gao L: DDX46 silencing inhibits cell proliferation by activating apoptosis and autophagy in cutaneous squamous cell carcinoma. Mol Med Rep 22: 4236-4242, 2020.

30. Liu Q, Dong J, Li J, Duan Y, Wang K, Kong Q and Zhang H: LINC01255 combined with BMI1 to regulate human mesenchymal stromal senescence and acute myeloid leukemia cell proliferation through repressing transcription of MCP-1. Clin Transl Oncol 23: 1105-1116, 2021.

31. White EJ, Brewer G and Wilson GM: Post-transcriptional control of gene expression by AUF1: Mechanisms, physiological targets, and regulation. Biochim Biophys Acta 1829: 680-688, 2013.

32. Yang Y, Wu J, Cai J, He Z, Yuan J, Zhu X, Li Y, Li M and Guan H: PSAT1 regulates cyclin D1 degradation and sustains proliferation of non-small cell lung cancer cells. Int J Cancer 136: E39-E50, 2015.
33. Duan W and Liu X: PSAT1 upregulation contributes to cell growth and cisplatin resistance in cervical cancer cells via regulating PI3K/AKT signaling pathway. Ann Clin Lab Sci 50: $512-518,2020$

34. Lu D, Di S, Zhuo S, Zhou L, Bai R, Ma T, Zou Z, Chen C, Sun M, Tang J, et al: The long noncoding RNA TINCR promotes breast cancer cell proliferation and migration by regulating OAS1. Cell Death Discov 7: 41, 2021

35. Suh YS, Yeom E, Nam JW, Min KJ, Lee J and Yu K Methionyl-tRNA synthetase regulates lifespan in Drosophila. Mol Cells 43: 304-311, 2020.

36. Jin Q, Liu G, Wang B, Li S, Ni K, Wang C, Ren J, Zhang S and Dai Y: High methionyl-tRNA synthetase expression predicts poor prognosis in patients with breast cancer. J Clin Pathol 73: 803-812, 2020

37. Wu MF, Stachon T, Langenbucher A, Seitz B and Szentmáry N: Effect of amniotic membrane suspension (AMS) and amniotic membrane homogenate $(\mathrm{AMH})$ on human corneal epithelial cell viability, migration and proliferation in vitro. Curr Eye Res 42: 351-357, 2017.

38. PLOS ONE Editors: Retraction: SPARC overexpression inhibits cell proliferation in neuroblastoma and is partly mediated by tumor suppressor protein PTEN and AKT. PLoS One 15: $\mathrm{e} 0228246,2020$.

39. Zhen J, Zhang H, Dong H and Tong X: miR-9-3p inhibits glioma cell proliferation and apoptosis by directly targeting FOXG1. Oncol Lett 20: 2007-2015, 2020.

40. Cheng Z, Zhang Y, Tian Y, Chen Y, Ding F, Wu H, Ji Y and Shen M: Cyr61 promotes Schwann cell proliferation and migration via $\alpha v \beta 3$ integrin. BMC Mol Cell Biol 22: 21, 2021.

41. Gui L, Zhu YW, Xu Q, Huang JJ, Hua P, Wu GJ, Lu J, Ni JB, Tang $\mathrm{H}$ and Zhang LL: RNA interference-mediated downregulation of phospholipid scramblase 1 expression in primary liver cancer in vitro. Oncol Lett 20: 361, 2020.

42. Held-Feindt J, Hattermann K, Knerlich-Lukoschus F, Mehdorn HM and Mentlein R: SP100 reduces malignancy of human glioma cells. Int J Oncol 38: 1023-1030, 2011.

43. Dang T, Modak C, Meng X, Wu J, Narvaez R and Chai J: CCN1 induces apoptosis in esophageal adenocarcinoma through p53-dependent downregulation of survivin. J Cell Biochem 120: 2070-2077, 2018

44. Wang R, Li KM, Zhou CH, Xue JL, Ji CN and Chen JZ: Cdc20 mediates D-box-dependent degradation of Sp100. Biochem Biophys Res Commun 415: 702-706, 2011

45. Verginelli F, Perin A, Dali R, Fung KH, Lo R, Longatti P, Guiot MC, Del Maestro RF, Rossi S, di Porzio U, et al: Transcription factors FOXG1 and Groucho/TLE promote glioblastoma growth. Nat Commun 4: 2956, 2013.

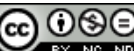

This work is licensed under a Creative Commons Attribution-NonCommercial-NoDerivatives 4.0 International (CC BY-NC-ND 4.0) License. 